\title{
Cognitive profiles in childhood and adolescence differ between adult psychotic and affective symptoms: a prospective birth cohort study
}

\author{
S. Koike ${ }^{1,2,3 *}$, J. Barnett ${ }^{4,5}$, P. B. Jones ${ }^{4,6}$ and M. Richards ${ }^{1}$ \\ ${ }^{1}$ MRC Unit for Lifelong Health and Ageing at UCL, 33 Bedford Place, London WC1B 5JU, UK \\ ${ }^{2}$ University of Tokyo Institute for Diversity \& Adaptation of Human Mind (UTIDAHM), 3-8-1 Komaba, Meguro-ku, Tokyo 153-8902, Japan \\ ${ }^{3}$ Center for Evolutionary Cognitive Sciences, Graduate School of Arts and Sciences, The University of Tokyo, 3-8-1 Komaba, Meguro-ku, Tokyo 153- \\ 8902, Japan \\ ${ }^{4}$ Department of Psychiatry, University of Cambridge, Cambridge CB2 OSZ, UK \\ ${ }^{5}$ Cambridge Cognition Ltd, Cambridge CB25 9TU, UK \\ ${ }^{6}$ CAMEO, Cambridgeshire \& Peterborough NHS Foundation Trust, Cambridge CB21 5EF, UK
}

Background. Differences between verbal and non-verbal cognitive development from childhood to adulthood may differentiate between those with and without psychotic symptoms and affective symptoms in later life. However, there has been no study exploring this in a population-based cohort.

Method. The sample was drawn from the MRC National Survey of Health and Development, and consisted of 2384 study members with self-reported psychotic experiences and affective symptoms at the age of 53 years, and with complete cognitive data at the ages of 8 and 15 years. The association between verbal and non-verbal cognition at age 8 years and relative developmental lag from age 8 to 15 years, and both adult outcomes were tested with the covariates adjusted, and mutually adjusted for verbal and non-verbal cognition.

Results. Those with psychotic experiences [thought interference $(n=433)$, strange experience $(n=296)$, hallucination $(n=88)$ ] had lower cognition at both the ages of 8 and 15 years in both verbal and non-verbal domains. After mutual adjustment, lower verbal cognition at age 8 years and greater verbal developmental lag were associated with higher likelihood of psychotic experiences within individuals, whereas there was no association between non-verbal cognition and any psychotic experience. In contrast, those with case-level affective symptoms $(n=453)$ had lower non-verbal cognition at age 15 years, and greater developmental lag in the non-verbal domain. After adjustment, lower non-verbal cognition at age 8 years and greater non-verbal developmental lag were associated with higher risk of case-level affective symptoms within individuals.

Conclusions. These results suggest that cognitive profiles in childhood and adolescence differentiate psychiatric disease spectra.

Received 15 June 2016; Revised 25 January 2017; Accepted 26 January 2017; First published online 9 October 2017

Key words: Anxiety, birth cohort studies, cognitive development, depression, schizophrenia.

\section{Introduction}

Epidemiological studies show that lower cognition in childhood is associated with the manifestation of schizophrenia (Jones et al. 1994; Cannon et al. 2002b; Zammit et al. 2004; Koenen et al. 2009; Welham et al. 2009; Reichenberg et al. 2010; Khandaker et al. 2011). This is in line with clinical case-control studies for first-episode psychosis as well as clinical high risk for psychosis (Fusar-Poli et al. 2012; Giuliano et al. 2012).

* Address for correspondence: S. Koike, M.D., Ph.D., Center for Evolutionary Cognitive Sciences, Graduate School of Art and Sciences, The University of Tokyo, 3-8-1 Komaba, Meguro-ku, Tokyo 153-8902, Japan.

(Email: skoike-tky@umin.ac.jp)
Lower childhood cognitive function is also observed in people with self-reported psychotic experiences (Horwood et al. 2008; Barnett et al. 2012; Gur et al. 2014; Khandaker et al. 2014, Nishida et al. 2014), suggesting that investigating the broader psychosis spectrum may help to understand the pathophysiology of schizophrenia. However, cognitive impairment in childhood also occurs with other psychiatric disorders such as major depression and self-report affective symptoms, suggesting common risk pathways (van Os et al. 1997; Zammit et al. 2004; Hatch et al. 2007; Koenen et al. 2009; Meier et al. 2014; Rock et al. 2014; Trivedi \& Greer, 2014).

Some longitudinal epidemiological studies have suggested that a developmental deficit in childhood (Reichenberg et al. 2010) or a developmental lag

This is an Open Access article, distributed under the terms of the Creative Commons Attribution licence (http://creative commons.org/licenses/by/4.0/), which permits unrestricted re-use, distribution, and reproduction in any medium, provided the original work is properly cited. 
(delay of typical development) through adolescence (Fuller et al. 2002; MacCabe et al. 2013) in verbal cognition differentiates those with schizophrenia from healthy participants, whereas verbal developmental lag does not appear to differentiate affective disorders (Cannon et al. 2006; Reichenberg et al. 2010). The former is also observed in children with dyslexia who are likely to have psychotic experiences in adolescence (Khandaker et al. 2014). Although still controversial (Carrion et al. 2011; Lin et al. 2013), clinical high-risk studies for psychosis showed that lower scores in verbal memory and verbal fluency tests could predict the later onset of psychosis (Giuliano et al. 2012), suggesting that developmental deficits and/or lag in verbal cognition play a key role in the emergence of psychotic symptoms. In contrast, epidemiological studies showed that people with affective symptoms have no specific developmental deficit and lag pattern in childhood (Reichenberg et al. 2010) and adulthood (Meier et al. 2014) in verbal and non-verbal cognition. Examining deficits and adolescent developmental lag in verbal and non-verbal cognition, and withinindividual cognitive discrepancy between the two cognitive domains between those with and without psychological symptoms in later life would test the specificity of the neurocognitive (and thus neurodevelopmental) model of psychosis and depression spectra (Fig. 1) (van Os, 2013). However, to the best of our knowledge, there has been no prospective cohort study that has examined whether relative verbal cognitive developmental lag in the general population is associated with adult psychotic experiences, and, conversely, whether non-verbal lag is associated with adult affective symptoms.

The Medical Research Council (MRC) National Survey of Health and Development (NSHD) is one of the longest continuously followed prospective birth cohort studies in the world, beginning in 1946 in England, Scotland and Wales (Wadsworth et al. 2006). Studies based on this cohort have reported cognitive delay in clinical cases of schizophrenia (Jones et al. 1994) and depression (van Os et al. 1997), as well as broader spectra of psychotic experiences (Barnett et al. 2012; Nishida et al. 2014) and affective symptoms (Hatch et al. 2007). Although symptoms in self-report questionnaires might have a different distribution to those from clinical interviews, the former are likely to be more representative at the general population level with broader disease spectra. In the present study, we extended this by examining psychosis and depression spectra in relation to deficits and adolescent developmental lag in verbal and nonverbal cognition. We hypothesized that a specific developmental deficit and lag in verbal cognition is associated with psychotic experiences in adulthood, but not with affective symptoms.

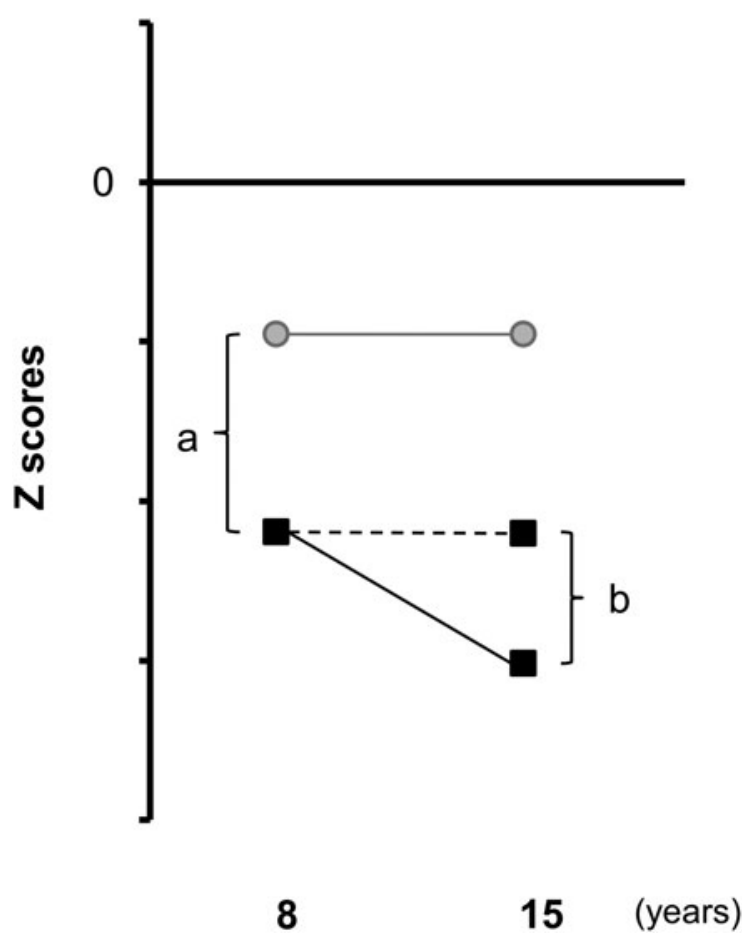

Fig. 1. Example of cognitive development in the two domains. Cognitive development using Z-scores of the two domains in this study is illustrated. Gap a indicates cognitive discrepancy between the two domains at age 8 years. Gap b indicates relative cognitive lag during adolescence, hypothesizing no difference in Z-scores through development within an individual (dashed line). Black line and square dots represent verbal cognition, and grey line and circle dots represent non-verbal cognition.

\section{Method}

\section{Participants}

The NSHD is one of the longest large prospective cohort studies, originally consisting of 5362 randomly selected children from all single births within marriage during 1 week in March 1946 in England, Scotland and Wales. Of the original sample, 3820 participants had complete cognitive data at ages 8 and 15 years. When study members were aged 53 years, they reported psychotic experiences and depressive symptoms in self-reported questionnaires. Of 3035 participants who were interviewed at that age, 2923 and 2920 participants provided information on psychotic experiences and affective symptoms (see below). Reasons for non-response at age 53 years were 469 deaths, 640 permanent refusals, 580 non-residents and 638 failures to contact. However, the responding participants were broadly representative of the national population of similar age (Wadsworth et al. 2003). Finally, one or more adult psychiatric outcomes were available for 2384 study members with complete cognitive data. Those with complete data were more 
likely to be female, had heavier birth weight, and higher cognition at ages 8 and 15 years than those with missing data $(p<0.05)$.

Ethical approval for this study was obtained from the North Thames Multicentre Research Ethics Committee. All participants gave written informed consent.

\section{Measures}

\section{Psychological symptoms in adulthood}

Psychotic experiences were self-reported over the previous 12 months at age 53 years using the five-item version of the Psychosis Screening Questionnaire (PSQ) (Bebbington \& Nayani, 1995; Johns et al. 2004) including three delusional (thought interference, persecution, and strange experiences), one hallucinatory and one hypomanic questions. All items were answered as either 'no', 'unsure' or 'yes'. In the original PSQ each of these questions has at least one follow-up question to elicit further detail; however, due to time constraints these were not asked in the NSHD. We therefore defined 'yes' for each leading question as the existence of a symptom and the other responses as negative. As in a previous study using this cohort (Nishida et al. 2014), more than half of the respondents gave a positive response to the hypomania item 'Have there been times when you felt very happy without a break for days on end?'. Further, 643 respondents $(22.0 \%)$ rated themselves as 'unsure' on this item. In addition, there has been a biological and cognitive debate over whether mania and hypomania should be included within the psychosis or affective spectrum (Trotta et al. 2015; Bora, 2016). Therefore, consistent with the previous study, we did not use this item in the analysis.

Self-reported affective symptoms were measured at age 53 years using the 28-item version of the General Health Questionnaire (GHQ-28) (Goldberg \& Hillier, 1979). Each item was scored using a four-level Likert scale for total symptom score, and was recoded into 0-0-1-1 for deriving a diagnostic risk threshold ('caseness'). This threshold was defined as a score of 5 or more on this summed recoded score (range 0-28), which was validated using the Clinical Interview Schedule (Goldberg \& Hillier, 1979). The GHQ-28 consists of four components (somatic symptoms, anxiety and insomnia, social dysfunction, and severe depression) (Goldberg \& Hillier, 1979). To test possible differential effects of these types of affective symptoms, we set thresholds for each subscale at the top $10 \%$ score, which matched the prevalence of GHQ-28 caseness and that of least one subscale.

\section{Cognition in childhood and adolescence}

Cognition at age 8 years was measured at school using three verbal tests (reading comprehension, word reading, and vocabulary) and one non-verbal test (picture intelligence, which consisted of three tasks: odd-one out, sequence detection and abstract reasoning) devised by the National Foundation for Educational Research (Pigeon, 1964). Cognition at age 15 years was measured at school using three verbal tests (Watts Vernon sentence completion, mathematics, and the verbal section of the Alice Heim Group Ability Test), and one non-verbal test (reasoning) using the non-verbal section of the Alice Heim Group Ability Test (Heim, 1970). The non-verbal tests at both ages had some similarity with the matrix reasoning and picture concepts subtests of the Wechsler Intelligence Scale for Children (Kaplan \& Saccuzzo, 2013). Non-verbal cognition also involves processing speed and is thought to be less dependent on educational and cultural learning than verbal cognition (Kaplan \& Saccuzzo, 2013). As verbal and non-verbal cognition was measured by different tests at ages 8 and 15 years, we calculated standardized verbal and non-verbal scores at both ages by summing each standardized score. We also calculated a relative developmental lag score for verbal and non-verbal cognition as the relevant $Z$-score at age 15 years minus corresponding $Z$-score at 8 years; thus a negative value represents relative decrease in scores from age 8 to 15 years, i.e. increasing developmental lag with respect to peers (Fig. 1). The ages 8 and 15 years were, respectively, the earliest and latest that cognition was tested during development. Thus this interval maximized the potential for capturing any effect of cognitive lag.

\section{Potential confounding variables}

Based on previous studies with this cohort the following potential confounders were selected: sex, birth weight, birth order, mother's education, and social class of origin (at age 11 years or, if this was unknown, at age 4 or 15 years).

Mother's education was dichotomized into primary only or above, and family social class was classified into three categories (professional or intermediate, skilled non-manual, and skilled manual or unskilled).

\section{Statistical analysis}

We initially used $\chi^{2}$ tests and $t$ tests, as appropriate, to test for differences between the independent variables in those with and without a positive response on the adult mental health outcomes [each individual PSQ item (all coded as positive or negative) or GHQ-28 'caseness']. Multivariable logistic regression was then used to test associations between childhood and adolescent cognition and the outcomes, using verbal and non-verbal cognition at age 8 years and verbal and non-verbal developmental lag from age 8 to 15 years 
as independent variables. Outcomes were coded as separate for each PSQ item and for GHQ-28 caseness. We also tested whether cognitive characteristics were associated with the number of positive PSQ responses using multivariate regression analysis. As several participants who reported the existence of psychotic experiences also had affective symptoms (53.9\%), we also grouped into psychotic experience only (PE only), affective symptoms only (AFF only), and both, with each of these groups compared with those with neither psychotic experience nor affective symptoms (no symptom) as the reference category. In addition, we compared the PE-only group with the AFF-only group to directly investigate differences in cognitive profiles. In this second analysis, psychotic experience presence was defined as one or more psychotic experience items of strange experience and hallucination, because previous studies showed a relatively low degree of positive responses that could represent more definitive characteristics of a psychosis spectrum (Johns et al. 2004; Nishida et al. 2014). For all multivariable models, we mutually adjusted verbal and non-verbal cognition. In addition, to test for possible synergy between verbal and non-verbal cognitive domains increasing the severity of outcomes, we tested interactions between verbal and non-verbal cognition at age 8 years and lag from age 8 to 15 years. Since verbal and non-verbal cognition are positively correlated (age 8 years: $r=0.57, p<0.001$; age 15 years: $r=0.61$, $p<0.001$ ), we tested multicollinearity using variance inflation factors (VIF) for mutual adjustment of cognitive variables. No collinearity was defined by less than 10 of each VIF value for the independent variable.

Statistical significance was set at 0.05 , and all analyses were conducted using SPSS Statistics 22 (IBM Corp., USA).

\section{Results}

Overall, the no-symptom group had stable Z-transformed cognitive scores from age 8 to 15 years in verbal and non-verbal domains (Table 1).

\section{Associations with adult psychotic experiences}

The rates of positive respondents for each psychotic experience items varied between $3.7 \%$ and $21.4 \%$, 'hallucination' was the lowest and 'persecution' the highest (Table 1). In comparing those with and without each psychotic experience, those with lower cognition in at least one domain were more likely to have psychotic experiences for all PSQ items at age 53 years except 'persecution'. These associations remained significant after adjusting for confounding variables (online Supplementary Table S2).
After mutual adjustment for verbal and non-verbal cognitive variables (maximum VIF $=2.2$ ), lower verbal cognition at age 8 years was associated with higher likelihood of each PSQ symptom except for 'persecution', whereas no significant association between non-verbal cognition and any of these psychotic experiences was evident (Table 2). In addition, greater verbal developmental lag from age 8 to 15 years was associated with higher likelihood of the psychotic experiences 'strange experience' and 'hallucination', whereas, again, there was no association between non-verbal lag and any of these psychotic experiences. In sum, those with relatively lower verbal cognition in childhood and adolescent verbal developmental lag, which resulted in wider cognitive discrepancy with relatively lower verbal cognition during adolescence, were more likely to have psychotic experiences (Fig. 2a).

\section{Associations with adult affective symptoms}

For case-level affective symptoms, 19.1\% (453/2376) participants were identified as cases. Frequencies of case-level somatic symptoms, anxiety and insomnia, social dysfunction and severe depression were 180 (7.6\%), $214(9.0 \%), 219(9.2 \%)$ and 182 (7.7\%), respectively (online Supplementary Table S1). There were $450(19.0 \%)$ study members with case-level symptoms for at least one subscale. Those with case-level symptoms had lower non-verbal cognition at age 15 years, and greater developmental lag in the non-verbal domain (Table 1). For verbal cognition, however, there was no association between those with and without affective symptoms.

After mutually adjusting for cognitive variables, lower non-verbal cognition at age 8 years and greater non-verbal developmental lag were associated with higher risk of GHQ-28 caseness (Table 2). There was no association between verbal cognitive scores and GHQ-28 caseness. These trends were similar for GHQ-28 subscore cases (online Supplementary Table S2). Multivariate regression analysis also showed that lower verbal cognition at age 8 years was associated with the number of positive responses in the PSQ at age 53 years $(B=-0.090$, s.E. $=0.033, p=0.007)$. In sum, those with relatively lower non-verbal cognition in childhood and adolescent non-verbal developmental lag, which resulted in wider cognitive discrepancy with relatively lower non-verbal cognitions, were more likely to have affective symptoms (Fig. 2b).

\section{Associations with adult psychotic experiences and affective symptoms}

Sample sizes for the grouped outcomes were 194 for PE only $(8.2 \%), 315$ for AFF only $(13.3 \%), 138$ for 
Table 1. Cognitive characteristics in childhood and adolescence on each psychotic experience and affective symptoms

\begin{tabular}{|c|c|c|c|c|c|c|c|c|c|c|c|c|}
\hline & \multicolumn{10}{|l|}{ PSQ } & \multirow{2}{*}{\multicolumn{2}{|c|}{$\begin{array}{l}\text { GHQ-28 } \\
\text { Affective symptoms }{ }^{\mathrm{a}}\end{array}$}} \\
\hline & \multicolumn{2}{|c|}{ Thought interference } & \multicolumn{2}{|l|}{ Persecution } & \multicolumn{2}{|c|}{ Strange experience } & \multicolumn{2}{|l|}{ Hallucination } & \multicolumn{2}{|c|}{ Any of psychotic experiences } & & \\
\hline & No or unsure & Yes & No or unsure & Yes & No or unsure & Yes & No or unsure & Yes & No or unsure & Yes & Non-case & Caseness \\
\hline $\begin{array}{l}\text { Participants, } \\
n(\%)\end{array}$ & $1945(81.8)$ & $433(18.2)$ & $1870(78.6)$ & $508(21.4)$ & $2083(87.6)$ & $296(12.4)$ & $2289(96.3)$ & $88(3.7)$ & $1607(67.5)$ & $772(32.5)$ & $1913(80.9)$ & $453(19.1)$ \\
\hline Male, $n(\%)$ & $959(82.7)$ & $200(17.3)$ & $905(78.2)$ & $253(21.8)$ & $1001(86.4)$ & $158(13.6)$ & 1105 (95.4) & $53(4.6)^{*}$ & $772(66.6)$ & 387 (33.4) & 998 (86.6) & $154(13.4)^{* * * *}$ \\
\hline \multicolumn{13}{|l|}{$\begin{array}{l}\text { Cognition at age } \\
8 \text { years }\end{array}$} \\
\hline Verbal domain & $0.10(0.99)$ & $-0.14(0.92)^{* * * *}$ & $0.07(0.99)$ & $0.00(0.97)$ & $0.07(0.98)$ & $-0.09(0.99)^{* *}$ & $0.07(0.98)$ & $-0.29(0.96)^{* * * *}$ & $0.11(0.99)$ & $-0.06(0.95)^{* * *}$ & $0.06(0.98)$ & $0.05(0.95)$ \\
\hline $\begin{array}{l}\text { Non-verbal } \\
\text { domain }\end{array}$ & $0.11(0.94)$ & $-0.07(1.01)^{* * *}$ & $0.08(0.95)$ & $0.03(0.98)$ & $0.09(0.94)$ & $-0.07(1.05)^{*}$ & $0.08(0.95)$ & $-0.24(1.08)^{* *}$ & $0.11(0.93)$ & $-0.00(1.00)^{* *}$ & $0.09(0.95)$ & $0.04(0.93)$ \\
\hline \multicolumn{13}{|l|}{$\begin{array}{l}\text { Cognition at age } \\
15 \text { years }\end{array}$} \\
\hline Verbal domain & $0.12(0.96)$ & $-0.05(0.89)^{* * *}$ & $0.10(0.95)$ & $0.05(0.95)$ & $0.12(0.94)$ & $-0.14(0.98)^{* * *}$ & $0.10(0.94)$ & $-0.32(1.00)^{* * *}$ & $0.13(0.95)$ & $0.00(0.94)^{* *}$ & $0.10(0.94)$ & $0.06(0.95)$ \\
\hline $\begin{array}{l}\text { Non-verbal } \\
\text { domain }\end{array}$ & $0.09(0.94)$ & $-0.06(0.95)^{* *}$ & $0.07(0.93)$ & $0.03(1.00)$ & $0.08(0.93)$ & $-0.06(1.05)^{*}$ & $0.07(0.94)$ & $-0.14(1.15)$ & $0.09(0.93)$ & $-0.00(0.98)^{*}$ & $0.10(0.93)$ & $-0.10(0.97)^{* * *}$ \\
\hline \multicolumn{13}{|l|}{$\begin{array}{l}\text { Developmental } \\
\text { lag from age } 8 \\
\text { to } 15 \text { years }\end{array}$} \\
\hline Verbal domain & $0.024(0.702)$ & $0.093(0.682)$ & $0.033(0.703)$ & $0.051(0.683)$ & $0.048(0.703)$ & $-0.048(0.662)^{*}$ & $0.038(0.702)$ & $-0.026(0.611)$ & $0.023(0.702)$ & $0.064(0.690)$ & $0.045(0.706)$ & $0.009(0.667)$ \\
\hline $\begin{array}{l}\text { Non-verbal } \\
\text { domain }\end{array}$ & $-0.019(1.015)$ & $0.016(1.118)$ & $-0.016(1.027)$ & $0.000(1.062)$ & $-0.016(1.030)$ & $0.008(1.067)$ & $-0.017(1.030)$ & $0.098(1.136)$ & $-0.020(1.005)$ & $0.002(1.093)$ & $0.019(1.042)$ & $-0.142(0.980)^{* *}$ \\
\hline
\end{tabular}

Data are given as mean (standard deviation) unless otherwise indicated.

PSQ, Psychosis Screening Questionnaire; GHQ-28, 28-item version of the General Health Questionnaire.

Value significantly different from the no or unsure participants: ${ }^{*} p<0.05,{ }^{* *} p<0.01,{ }^{* * *} p<0.001$ (by $\chi^{2}$ test for sex, and $t$ test for the other variables).

${ }^{a}$ Differences in existence of GHQ-28 subscale cases are shown in online Supplementary Table S1.

${ }^{\mathrm{b}}$ Differences in Z-scores from age 8 to 15 years for verbal and non-verbal domains (score at age 15 years -8 years). A positive score represents an increase of developmental lag from the mean. 
Table 2. Association of cognitive characteristics in childhood and adolescence with each psychotic experience and affective symptoms ${ }^{\mathrm{a}}$

\begin{tabular}{|c|c|c|c|c|c|c|}
\hline \multirow[b]{2}{*}{ Independent variables } & \multicolumn{5}{|l|}{ PSQ } & \multirow{2}{*}{$\begin{array}{l}\text { GHQ-28 } \\
\\
\text { Affective symptoms } \\
(n=453 \text { v. 1913) }\end{array}$} \\
\hline & $\begin{array}{l}\text { Thought interference } \\
(n=433 v .1945)\end{array}$ & $\begin{array}{l}\text { Persecution } \\
(n=508 v .1870)\end{array}$ & $\begin{array}{l}\text { Strange experience } \\
(n=296 v .2083)\end{array}$ & $\begin{array}{l}\text { Hallucination } \\
(n=88 v .2289)\end{array}$ & $\begin{array}{l}\text { Any of psychotic } \\
\text { experiences } \\
(n=772 v .1607)\end{array}$ & \\
\hline \multicolumn{7}{|l|}{ Cognition at age 8 years } \\
\hline Verbal domain & $0.81(0.68-0.97)^{*}$ & $0.92(0.78-1.09)$ & $0.79(0.64-0.97)^{*}$ & $0.55(0.38-0.80)^{* *}$ & $0.82(0.70-0.94)^{* *}$ & $1.05(0.88-1.26)$ \\
\hline Non-verbal domain & $0.91(0.76-1.10)$ & $1.00(0.84-1.19)$ & $0.99(0.80-1.22)$ & $1.02(0.70-1.47)$ & $0.99(0.85-1.15)$ & $0.77(0.64-0.93)^{* *}$ \\
\hline \multicolumn{7}{|c|}{ Developmental lag from age 8 to 15 years } \\
\hline Verbal domain & $1.07(0.88-1.31)$ & $0.99(0.83-1.20)$ & $0.71(0.56-0.90)^{* *}$ & $0.52(0.34-0.79)^{* *}$ & $0.98(0.83-1.15)$ & $1.11(0.91-1.36)$ \\
\hline Non-verbal domain & $0.93(0.80-1.08)$ & $1.00(0.87-1.14)$ & $1.04(0.88-1.24)$ & $1.23(0.91-1.67)$ & $0.98(0.87-1.10)$ & $0.74(0.64-0.86)^{* * *}$ \\
\hline
\end{tabular}

Data are given as odds ratio (95\% confidence interval).

PSQ, Psychosis Screening Questionnaire; GHQ-28, 28-item version of the General Health Questionnaire.

${ }^{a}$ This model was adjusted by the confounding variables of sex, birth weight, birth order, mother's education, and childhood social class, and the independent variables of cognition at age 8 years and developmental lag from age 8 to 15 years in verbal and non-verbal domains. Unadjusted and other adjusted models, and models for GHQ-28 subscale cases are shown in online Supplementary Table S2.

Significant coefficient: ${ }^{*} p<0.05,{ }^{* *} p<0.01,{ }^{* * *} p<0.001$. 


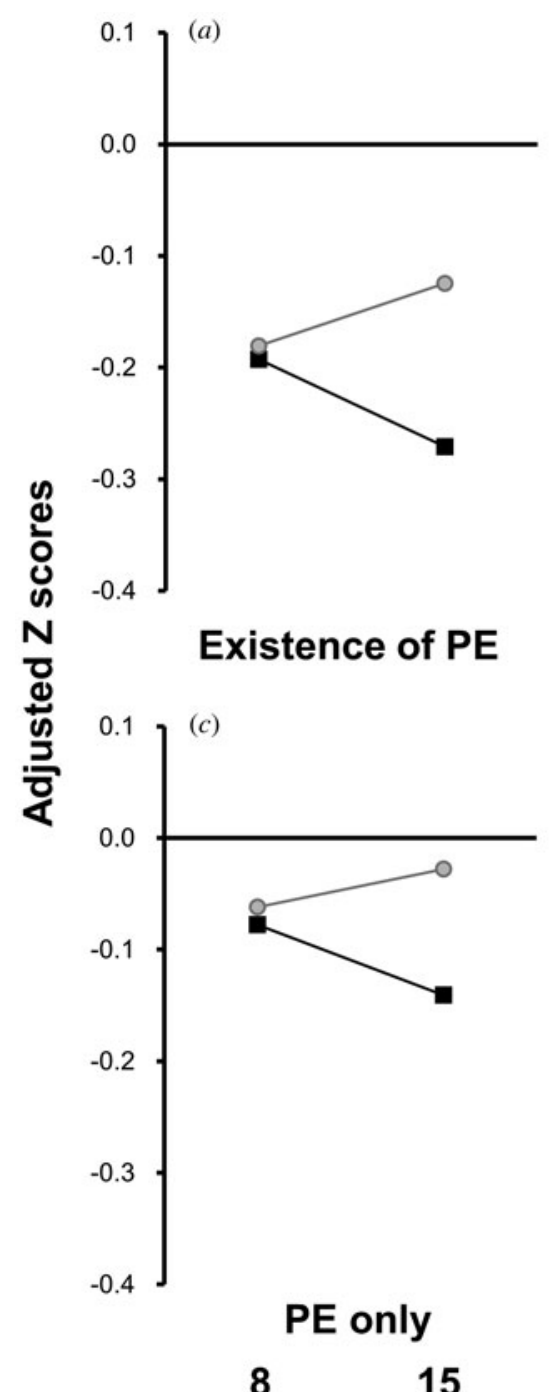

8 $\downarrow$

(b)

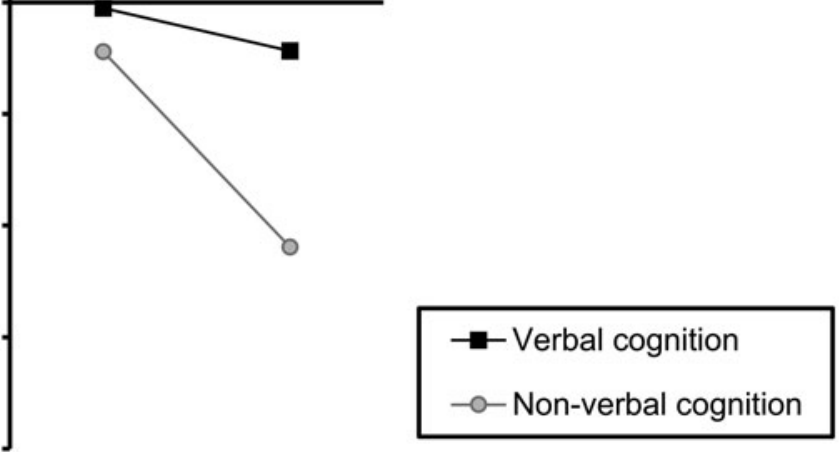

Existence of AFF

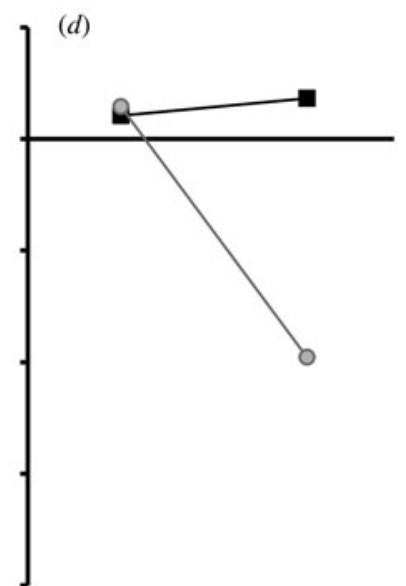

AFF only

8

15

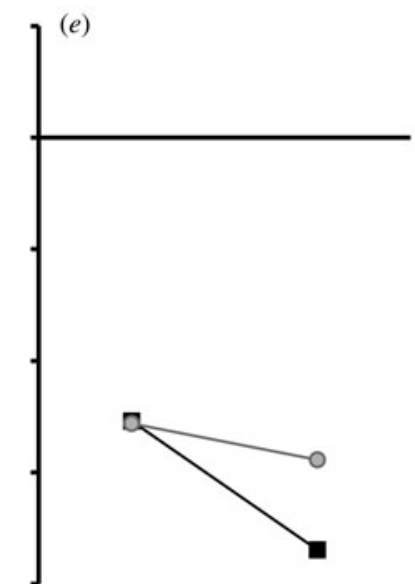

Both

815 (years)

Fig. 2. Cognitive development patterns by existence of psychotic experiences (PE) and affective symptoms (AFF). $(a, b)$ Characteristics of cognitive scores in those with PE (and AFF) are illustrated using adjusted Z-transformed scores for sex and mother's education, and for those without PE (and AFF) as zero. (c-e) Characteristics of three different subgroups (PE only, AFF only, and both symptoms) are also illustrated using adjusted Z-transformed scores, with the no-symptom group as zero.

both $(5.8 \%)$ and 1714 for the no-symptom group $(72.6 \%)$. In logistic regression models with the no-symptom group as the reference, the AFF-only group showed the characteristics of the cognitive discrepancy; a smaller verbal developmental lag (i.e. relatively better development in verbal cognition), lower non-verbal cognition at age 8 years, and a greater nonverbal developmental lag (Table 3). These trends are illustrated in Fig. $2 c-e$, particularly the non-verbal developmental lag in the AFF-only group and greater cognitive discrepancy (non-verbal < verbal), while the PE-only group had a developmental lag in verbal cognition and greater cognitive discrepancy (verbal< non-verbal).

\section{Interactions between verbal and non-verbal cognition on adult psychological symptoms}

When we added interactions between verbal and nonverbal cognitive domains at age 8 years and developmental lags from age 8 to 15 years to the mutually adjusted models, we found a significant interaction between verbal and non-verbal cognition at age 8 years on 'thought interference' at age 53 years [odds ratio $(\mathrm{OR})=0.87$, 95\% confidence interval (CI) 0.78 $0.99, p=0.029)$, with the association between lower verbal cognition at age 8 years and PE similar to the above results. Thus, greater cognitive discrepancy (verbal< non-verbal) in addition to lower verbal cognition at 
Table 3. Cognitive characteristics in childhood and adolescence by existence of psychotic experience and/or affective symptoms ${ }^{\mathrm{a}}$

\begin{tabular}{lllll}
\hline Independent variables & $\begin{array}{l}\text { PE only }(n=194) v . \text { no } \\
\text { symptoms }(n=1714)\end{array}$ & $\begin{array}{l}\text { AFF only }(n=315) v . \text { no } \\
\text { symptoms }(n=1714)\end{array}$ & $\begin{array}{l}\text { Both }(n=138) v . \text { no } \\
\text { symptoms }(n=1714)\end{array}$ & $\begin{array}{l}\text { AFF only }(n=315) v . \\
\text { PE only }(n=194)\end{array}$ \\
\hline $\begin{array}{l}\text { Cognition at age } 8 \\
\text { years }\end{array}$ & & & & \\
$\quad$ Verbal domain & $0.79(0.61-1.02)$ & $1.21(0.98-1.49)$ & $0.72(0.53-0.97)^{*}$ & $0.64(0.45-0.91)^{*}$ \\
$\quad \begin{array}{l}\text { Non-verbal domain } \\
1.12(0.86-1.46)\end{array}$ & $0.73(0.58-0.91)^{* *}$ & $0.85(0.63-1.15)$ & $1.40(0.98-2.01)$ \\
$\begin{array}{l}\text { Developmental lag } \\
\text { from age 8 to 15 years }\end{array}$ & & & & $0.59(0.40-0.86)^{* *}$ \\
$\quad$ Verbal domain & $0.75(0.57-1.00)$ & $1.33(1.05-1.68)^{*}$ & $0.70(0.50-0.99)^{*}$ & $1.50(1.13-2.01)^{* *}$ \\
$\quad$ Non-verbal domain & $1.12(0.91-1.38)$ & $0.66(0.55-0.79)^{* * *}$ & $0.93(0.72-1.18)$ & \\
\hline
\end{tabular}

Data are given as odds ratio (95\% confidence interval).

PE, Psychotic experience; AFF, affective symptoms.

${ }^{\text {a }}$ Participants were grouped into PE-only (defined by the existence of one or more items of strange experience and hallucination), AFF-only, both, and no-symptom groups. This model was adjusted by the confounding variables of sex, birth weight, birth order, mother's education and childhood social class, and the independent variables of cognition at age 8 years and developmental lag from age 8 to 15 years in verbal and non-verbal domains. Unadjusted and other adjusted models are shown in online Supplementary Table S3.

Significant coefficient: ${ }^{*} p<0.05,{ }^{* *} p<0.01,{ }^{* * *} p<0.001$.

age 8 years was additively associated with adult 'thought interference'.

Compared with the 'no'-symptom group, the interaction between verbal and non-verbal cognitive lags from age 8 to 15 years was also significant in the 'both'symptom group $(\mathrm{OR}=0.68,95 \%$ CI $0.52-0.90, p=$ 0.007), with a similar effect to the above results of lower verbal cognition at age 8 years and greater verbal cognitive lag. Thus, greater discrepancy between verbal and non-verbal cognitive lags from age 8 to 15 years (verbal< non-verbal) was associated with the simultaneous existence of psychotic and affective symptoms at age 53 years.

\section{Discussion}

A population-representative prospective birth cohort study revealed a developmental verbal-non-verbal differentiation in regard to the presence of adult psychotic experiences and affective symptoms. Low verbal cognition at age 8 years and relative developmental lag in this domain between age 8 and 15 years were associated with psychotic experiences at age 53 years, independently of non-verbal cognition and confounding factors. In contrast, lower non-verbal cognition at age 8 years and relative developmental lag in this between age 8 and 15 years were associated with risk of common mental disorder in adulthood, as assessed by the GHQ-28 caseness threshold, independently of verbal cognition and the other covariates. Participants with case-level affective symptoms but no psychotic experience additionally had higher verbal cognition at age 8 years, and rather better verbal development between the ages of 8 and 15 years. To the best of our knowledge, this is the first investigation of its kind in a population-representative prospective birth cohort study.

Strengths of this study include a large populationbased prospective birth cohort, the availability of cognition objectively assessed in childhood and adolescence, and the availability of a wide range of potential confounders. In addition, since psychotic experiences are less common in midlife than in adolescence or early adulthood (van Os et al. 2009), these symptoms at age 53 years may be associated with more distinct cognitive profiles than those reported at a younger age (Nishida et al. 2014). The childhood and adolescent cognitive characteristics in those with adult psychotic or depressive symptoms are in line with previous clinical studies in patients and high-risk individuals, suggesting that the characteristics of cognitive development are common within each spectrum. Although some responses using self-report questionnaires are state-dependent, our results could deserve further exploration of the relationship between cognitive profiles and psychological outcomes.

However, several limitations should also be considered. Although demographic characteristics in the NSHD were representative of the population in the survey at age 53 years (Wadsworth et al. 2003), those lost to follow-up were more likely to be male, and had lower birth weight and lower cognition scores at ages 8 and 15 years. Lower childhood cognition and adult psychological symptoms are associated with attrition in cohort studies. Similar to the results from clinical studies of schizophrenia and major affective 
symptoms, people with psychotic experiences had higher all-cause mortality in a prospective study (Sharifi et al. 2015). As clinical studies have repeatedly shown these characteristics to be risk factors for schizophrenia, higher attrition for participants with cognitive discrepancy may have led to underestimation of effect size in the relationship between greater verbal developmental lag and psychotic experiences. Attrition of those with low childhood cognitive function may similarly have caused underestimation of effect sizes. However, we have no reason to believe that this would have altered the pattern of associations observed. Second, due to time constraints we did not ask the supplementary questions to each of the five leading PSQ questions, which resulted in some detail about psychotic features being lost. Third, self-report questionnaires may influence responses through misinterpretation or misreading of items, or by transient events. Although the present findings were consistent with clinical case-control studies of disease spectra, repeated measures and interview-based assessment may increase response validity. In addition, the PSQ scale was tested at an item level that may not have allowed sufficient validity, and also was not comparable with the way we used the GHQ-28 as a total score. However, a total score based on four items would not have similar psychometric properties to that based on a 28-item Likert scale. In any case, recent evidence suggests that positive self-reported psychotic experiences are multi-factorial (Therman \& Ziermans, 2016). Future research using threshold-level psychotic experiences is needed to confirm the present findings. Fourth, since detailed information for clinical treatment was not available, we were unable to consider whether participants with schizophrenia and major depression in remission were among those who were classed as negative for symptoms. However, this is unlikely to be a significant source of bias, and those with clinical symptoms are only a subgroup of those who self-report symptoms (Jones et al. 1994; van Os et al. 1997). Although 30 clinical cases of schizophrenia were identified in this cohort, only 11 of these cases completed the GHQ-28 at the 53-year survey (Jones et al. 1994). Thus we did not use this classification to test cognitive discrepancy in the psychosis spectrum compared with participants with affective symptoms. Consistent with this, a previous study using this cohort reported that psychotropic medication at age 43 years had little effect on the GHQ-28 score at age 53 years (Colman et al. 2008). Fifth, although comparisons with short-form intelligence tests suggest that nonverbal tests at age 8 and 15 years broadly represent non-verbal cognition (Christensen et al. 2007), processing speed as measured by, for example, the symbol coding and symbol search subtests was not assessed in this study. We previously showed a deficit of motor development in those who later developed schizophrenia and affective disorders (Jones et al. 1994; van Os et al. 1997); however, another cohort study did not show a developmental deficit in relation to digit symbol substitution, although a developmental lag through adolescence occurred in people with schizophrenia (Reichenberg et al. 2010; Meier et al. 2014). In addition, it is a possibility that the participants might use a verbal strategy when performing non-verbal cognitive tests in this study. Further studies are needed of non-verbal cognitive deficit and lag in relation to psychotic and depressive symptoms.

Although cognitive deficits in various domains are evident in patients with schizophrenia (Jones et al. 1994; Cannon et al. 2002b; Fuller et al. 2002; Zammit et al. 2004; Koenen et al. 2009; Welham et al. 2009; Reichenberg et al. 2010; Khandaker et al. 2011; Trotta et al. 2015; Bora, 2016) and individuals at high risk of psychosis (Carrion et al. 2011; Fusar-Poli et al. 2012; Giuliano et al. 2012; Lin et al. 2013), our results suggest that lower verbal performance is a specific characteristic of the psychosis spectrum. This is in line with another cohort study showing an inverse association between psychotic experiences and intelligence quotient (IQ), especially in verbal IQ after adjusting for non-verbal IQ (Horwood et al. 2008). Neuroimaging studies have also shown that individuals with clinical high risk for psychosis had similar brain volume reduction and function in the inferior frontal gyrus, a region responsible for language processing, to patients with first-episode psychosis and chronic schizophrenia (Koike et al. 2011; Iwashiro et al. 2012; Koike et al. 2016).

Of interest, a study using national register data showed that siblings of patients with schizophrenia were more likely to work in creative occupations (e.g. in those with scientific occupations) (Kyaga et al. 2011). This association was strengthened after controlling for overall cognition (Kyaga et al. 2011). Creativity is more linked to non-verbal cognitive ability than verbal cognition (Cerruti \& Wilkey, 2011), suggesting that a discrepancy towards higher non-verbal cognition may be associated with advantage in creative occupations. On the other hand, divergent thinking, related to creativity, is associated with thought disorder in schizophrenia, which is assumed to be derived from verbal cognitive impairment (Crow, 2008; Levy et al. 2010).

In contrast, participants with case-level affective symptoms had lower non-verbal cognition at age 8 years and greater relative developmental non-verbal lag, independently of verbal cognition and the other covariates. Consistent with previous studies (Cannon et al. 2002a; Koenen et al. 2009; Reichenberg et al. 2010), verbal cognition in those with affective symptoms was not different from that in participants 
without case-level affective symptoms, even without control for potential confounders, and was relatively high in those with co-morbid psychotic experiences. A meta-analysis suggests that non-verbal executive function, such as visual planning, spatial working memory, visual attention and visual pattern recognition memory, is one of the most prominent cognitive impairments in major depression (Rock et al. 2014; Trivedi \& Greer, 2014). In addition, these impairments are evident before clinical onset, and show little improvement during clinical course, suggesting a trait factor (Rock et al. 2014; Trivedi \& Greer, 2014). A recent neuroimaging study showed that a greater verbal-non-verbal discrepancy towards lower non-verbal function was associated with cortical thinning in the rostral part of the prefrontal cortex (Margolis et al. 2013), which is one of the regions responsible for executive functions (Badre \& D'Esposito, 2009) as well as major depression (Disner et al. 2011).

In conclusion, we found that people with adult psychotic experiences and depressive symptoms showed differential verbal-non-verbal cognitive profiles in childhood and adolescence. Our results suggest that these characteristics of cognitive development are common within each disease spectrum. Future prospective studies are needed at different stages across the spectra to elucidate how cognitive profiles are associated with the emergence of psychological symptoms.

\section{Supplementary material}

The supplementary material for this article can be found at https://doi.org/10.1017/S0033291717000393

\section{Acknowledgements}

The authors are grateful to NSHD study members for their continuing support. S.K. was supported by grants from the Japan Society for the Promotion of Science (JSPS) (KAKENHI grants no. 25870143 and 26118703) and the Strategic Young Researcher Overseas Visits Program for Accelerating Brain Circulation. M.R. is funded by the UK Medical Research Council (Unit Programme no. MC UU 12019/3). P.B.J. is supported by Wellcome Trust grants no. 095844/Z/11/Z and 088869/Z/09/Z; National Institute for Health Research grant no. RP-PG-0606-1335; and by the National Institute for Health Research (NIHR) Cambridge Biomedical Research Centre (BRC) and the NIHR Collaboration for Leadership in Applied Health Research \& Care (CLAHRC) East of England.

\section{Declaration of Interest}

P.B.J. has received research grant support from GlaxoSmithKline, a speaker's honorarium from Eli
Lilly, and is a co-inventor of patent PCT/GB2005/ 003279 (methods for assessing psychotic disorders). J.B. is an employee of Cambridge Cognition Ltd, and is a co-inventor of patent PCT/GB2005/003279 (methods for assessing psychotic disorders). S.K. and M.R. report no competing interests.

\section{References}

Badre D, D'Esposito M (2009). Is the rostro-caudal axis of the frontal lobe hierarchical? Nature Reviews Neuroscience 10, 659-669.

Barnett JH, McDougall F, Xu MK, Croudace TJ, Richards M, Jones PB (2012). Childhood cognitive function and adult psychopathology: associations with psychotic and non-psychotic symptoms in the general population. British Journal of Psychiatry 201, 124-130.

Bebbington P, Nayani T (1995). The Psychosis Screening Questionnaire. International Journal of Methods in Psychiatric Research 9, 139-145.

Bora E (2016). Differences in cognitive impairment between schizophrenia and bipolar disorder: considering the role of heterogeneity. Psychiatry and Clinical Neurosciences 70, 424-433.

Cannon M, Caspi A, Moffitt TE, Harrington H, Taylor A, Murray RM, Poulton R (2002a). Evidence for early-childhood, pan-developmental impairment specific to schizophreniform disorder: results from a longitudinal birth cohort. Archives of General Psychiatry 59, 449-456.

Cannon M, Jones PB, Murray RM (2002b). Obstetric complications and schizophrenia: historical and meta-analytic review. American Journal of Psychiatry 159, 1080-1092.

Cannon M, Moffitt TE, Caspi A, Murray RM, Harrington H, Poulton R (2006). Neuropsychological performance at the age of 13 years and adult schizophreniform disorder: prospective birth cohort study. British Journal of Psychiatry 189, 463-464.

Carrion RE, Goldberg TE, McLaughlin D, Auther AM, Correll CU, Cornblatt BA (2011). Impact of neurocognition on social and role functioning in individuals at clinical high risk for psychosis. American Journal of Psychiatry 168, 806-813.

Cerruti C, Wilkey E (2011). Verbal overshadowing and verbal facilitation in creative cognition. In Perspectives on Creativity (ed. L DellaPietra), pp. 178-187. Cambridge Scholars Publishing: Newcastle upon Tyne.

Christensen BK, Girard TA, Bagby RM (2007). Wechsler Adult Intelligence Scale-Third Edition short form for index and IQ scores in a psychiatric population. Psychological Assessment 19, 236-240.

Colman I, Croudace TJ, Wadsworth ME, Kuh D, Jones PB (2008). Psychiatric outcomes 10 years after treatment with antidepressants or anxiolytics. British Journal of Psychiatry 193, 327-331.

Crow TJ (2008). The 'big bang' theory of the origin of psychosis and the faculty of language. Schizophrenia Research 102, 31-52. 
Disner SG, Beevers CG, Haigh EA, Beck AT (2011). Neural mechanisms of the cognitive model of depression. Nature Reviews Neuroscience 12, 467-477.

Fuller R, Nopoulos P, Arndt S, O'Leary D, Ho BC, Andreasen NC (2002). Longitudinal assessment of premorbid cognitive functioning in patients with schizophrenia through examination of standardized scholastic test performance. American Journal of Psychiatry 159, 1183-1189.

Fusar-Poli P, Deste G, Smieskova R, Barlati S, Yung AR, Howes O, Stieglitz RD, Vita A, McGuire P, Borgwardt S (2012). Cognitive functioning in prodromal psychosis: a meta-analysis. Archives of General Psychiatry 69, 562-571.

Giuliano AJ, Li H, Mesholam-Gately RI, Sorenson SM, Woodberry KA, Seidman LJ (2012). Neurocognition in the psychosis risk syndrome: a quantitative and qualitative review. Current Pharmaceutical Design 18, 399-415.

Goldberg DP, Hillier VF (1979). A scaled version of the General Health Questionnaire. Psychological Medicine 9, 139-145.

Gur RC, Calkins ME, Satterthwaite TD, Ruparel K, Bilker WB, Moore TM, Savitt AP, Hakonarson H, Gur RE (2014). Neurocognitive growth charting in psychosis spectrum youths. JAMA Psychiatry 71, 366-374.

Hatch SL, Jones PB, Kuh D, Hardy R, Wadsworth ME, Richards M (2007). Childhood cognitive ability and adult mental health in the British 1946 birth cohort. Social Science and Medicine 64, 2285-2296.

Heim AW (1970). The AH4 Group Test of Intelligence. NFER-Nelson: Windsor.

Horwood J, Salvi G, Thomas K, Duffy L, Gunnell D, Hollis C, Lewis G, Menezes P, Thompson A, Wolke D, Zammit S, Harrison G (2008). IQ and non-clinical psychotic symptoms in 12-year-olds: results from the ALSPAC birth cohort. British Journal of Psychiatry 193, 185-191.

Iwashiro N, Suga M, Takano $Y$, Inoue H, Natsubori T, Satomura Y, Koike S, Yahata N, Murakami M, Katsura M, Gonoi W, Sasaki H, Takao H, Abe O, Kasai K, Yamasue H (2012). Localized gray matter volume reductions in the pars triangularis of the inferior frontal gyrus in individuals at clinical high-risk for psychosis and first episode for schizophrenia. Schizophrenia Research 137, 124-131.

Johns LC, Cannon M, Singleton N, Murray RM, Farrell M, Brugha T, Bebbington P, Jenkins R, Meltzer H (2004). Prevalence and correlates of self-reported psychotic symptoms in the British population. British Journal of Psychiatry 185, 298-305.

Jones P, Rodgers B, Murray R, Marmot M (1994). Child development risk factors for adult schizophrenia in the British 1946 birth cohort. Lancet 344, 1398-1402.

Kaplan RM, Saccuzzo DP (2013). Psychological Testing: Principles, Applications, and Issues, 8th edn. Wadsworth, Cengage Learning: Boston, MA.

Khandaker GM, Barnett JH, White IR, Jones PB (2011). A quantitative meta-analysis of population-based studies of premorbid intelligence and schizophrenia. Schizophrenia Research 132, 220-227.

Khandaker GM, Stochl J, Zammit S, Lewis G, Jones PB (2014). A population-based longitudinal study of childhood neurodevelopmental disorders, IQ and subsequent risk of psychotic experiences in adolescence. Psychological Medicine 44, 3229-3238.

Koenen KC, Moffitt TE, Roberts AL, Martin LT, Kubzansky L, Harrington H, Poulton R, Caspi A (2009). Childhood IQ and adult mental disorders: a test of the cognitive reserve hypothesis. American Journal of Psychiatry 166, 50-57.

Koike S, Satomura Y, Kawasaki S, Nishimura Y, Takano Y, Iwashiro N, Kinoshita A, Nagai T, Natsubori T, Tada M, Ichikawa E, Takizawa R, Kasai K (2016). Association between rostral prefrontal cortical activity and functional outcome in first-episode psychosis: a longitudinal functional near-infrared spectroscopy study. Schizophrenia Research 170, 304-310.

Koike S, Takizawa R, Nishimura $Y$, Takano $Y$, Takayanagi Y, Kinou M, Araki T, Harima H, Fukuda M, Okazaki Y, Kasai K (2011). Different hemodynamic response patterns in the prefrontal cortical sub-regions according to the clinical stages of psychosis. Schizophrenia Research 132, 54-61.

Kyaga S, Lichtenstein P, Boman M, Hultman C, Långström N, Landén M (2011). Creativity and mental disorder: family study of 300,000 people with severe mental disorder. British Journal of Psychiatry 199, 373-379.

Levy DL, Coleman MJ, Sung H, Ji F, Matthysse S, Mendell NR, Titone D (2010). The genetic basis of thought disorder and language and communication disturbances in schizophrenia. Journal of Neurolinguistics 23, 176.

Lin A, Yung AR, Nelson B, Brewer WJ, Riley R, Simmons M, Pantelis C, Wood SJ (2013). Neurocognitive predictors of transition to psychosis: medium- to long-term findings from a sample at ultra-high risk for psychosis. Psychological Medicine 43, 2349-2360.

MacCabe JH, Wicks S, Lofving S, David AS, Berndtsson A, Gustafsson JE, Allebeck P, Dalman C (2013). Decline in cognitive performance between ages 13 and 18 years and the risk for psychosis in adulthood: a Swedish longitudinal cohort study in males. JAMA Psychiatry 70, 261-270.

Margolis A, Bansal R, Hao X, Algermissen M, Erickson C, Klahr KW, Naglieri JA, Peterson BS (2013). Using IQ discrepancy scores to examine the neural correlates of specific cognitive abilities. Journal of Neuroscience 33, 1413514145.

Meier MH, Caspi A, Reichenberg A, Keefe RS, Fisher HL, Harrington H, Houts R, Poulton R, Moffitt TE (2014). Neuropsychological decline in schizophrenia from the premorbid to the postonset period: evidence from a population-representative longitudinal study. American Journal of Psychiatry 171, 91-101.

Nishida A, Xu KM, Croudace T, Jones PB, Barnett J, Richards M (2014). Adolescent self-control predicts midlife hallucinatory experiences: 40-year follow-up of a national birth cohort. Schizophrenia Bulletin 40, 1543-1551.

Pigeon DA (1964). Tests used in the 1954 and 1957 surveys. In The Home and the School (ed. JWB Douglas), appendix 1. Macgibbon \& Kee: London.

Reichenberg A, Caspi A, Harrington H, Houts R, Keefe RS, Murray RM, Poulton R, Moffitt TE (2010). Static and dynamic cognitive deficits in childhood preceding adult 
schizophrenia: a 30-year study. American Journal of Psychiatry 167, 160-169.

Rock PL, Roiser JP, Riedel WJ, Blackwell AD (2014). Cognitive impairment in depression: a systematic review and meta-analysis. Psychological Medicine 44, 2029-2040.

Sharifi V, Eaton WW, Wu LT, Roth KB, Burchett BM, Mojtabai R (2015). Psychotic experiences and risk of death in the general population: 24-27 year follow-up of the Epidemiologic Catchment Area study. British Journal of Psychiatry 207, 30-36.

Therman S, Ziermans TB (2016). Confirmatory factor analysis of psychotic-like experiences in a general population sample. Psychiatry Research 235, 197-199.

Trivedi MH, Greer TL (2014). Cognitive dysfunction in unipolar depression: implications for treatment. Journal of Affective Disorders 152-154, 19-27.

Trotta A, Murray RM, MacCabe JH (2015). Do premorbid and post-onset cognitive functioning differ between schizophrenia and bipolar disorder? A systematic review and meta-analysis. Psychological Medicine 45, 381-394.

van Os J (2013). The dynamics of subthreshold psychopathology: implications for diagnosis and treatment. American Journal of Psychiatry 170, 695-698.

van Os J, Jones P, Lewis G, Wadsworth M, Murray R (1997). Developmental precursors of affective illness in a general population birth cohort. Archives of General Psychiatry 54, 625-631.

van Os J, Linscott RJ, Myin-Germeys I, Delespaul P, Krabbendam L (2009). A systematic review and meta-analysis of the psychosis continuum: evidence for a psychosis proneness-persistence-impairment model of psychotic disorder. Psychological Medicine 39, 179-195.

Wadsworth M, Kuh D, Richards M, Hardy R (2006). Cohort profile: The 1946 National Birth Cohort (MRC National Survey of Health and Development). International Journal of Epidemiology 35, 49-54.

Wadsworth ME, Butterworth SL, Hardy RJ, Kuh DJ, Richards M, Langenberg C, Hilder WS, Connor M (2003). The life course prospective design: an example of benefits and problems associated with study longevity. Social Science and Medicine 57, 2193-2205.

Welham J, Isohanni M, Jones P, McGrath J (2009). The antecedents of schizophrenia: a review of birth cohort studies. Schizophrenia Bulletin 35, 603-623.

Zammit S, Allebeck P, David AS, Dalman C, Hemmingsson T, Lundberg I, Lewis G (2004). A longitudinal study of premorbid IQ score and risk of developing schizophrenia, bipolar disorder, severe depression, and other nonaffective psychoses. Archives of General Psychiatry 61, 354-360. 